\title{
Considering mutational meltdown as a potential SARS-CoV-2 treatment strategy
}

\author{
Jeffrey D. Jensen ${ }^{1} \cdot$ Michael Lynch ${ }^{1}$
}

Received: 1 April 2020 / Revised: 2 April 2020 / Accepted: 3 April 2020 / Published online: 6 April 2020

(c) The Genetics Society 2020

With the rapid global spread of SARS-CoV-2, tremendous efforts have been focused upon potential treatment strategies ( $\mathrm{Li}$ and De Clercq 2020). Evolutionary theory has an important role to play in this search, and we here discuss one potentially under-appreciated research avenue.

Within the field of population genetics, the phenomenon of mutational meltdown-in which a population may become extinct owing to the accumulation of deleterious mutations-has been well studied both theoretically and experimentally. The key to understanding this effect is a consideration of the efficacy of natural selection. Because there are many more ways to disrupt rather than to improve genomic function, the vast majority of new fitnessimpacting mutations are deleterious rather than beneficial. Thus, if mutation rates are increased, the result is a disproportionate excess of variants that are detrimental to the organism. Because natural selection will not be able to purge this input of deleterious mutations if the mutational pressure is sufficiently large, these variants may remain in the population and even reach fixation. This deleterious load further restricts the ability of natural selection to purge additional variants, allowing more deleterious mutations to accumulate and fix, and so on-a snowball effect that can result in the eventual loss of the population (i.e., mutational meltdown).

Lynch and Gabriel (1990) and Lynch et al. (1993) described this model, which is dependent on the carrying capacity of the population, the absolute population growth rate, the deleterious effect of mutations, and the deleterious mutation rate. Under this model, if the input of deleterious mutations is sufficiently high, the number of reproducing individuals will decline. Though the model of lethal

Jeffrey D. Jensen

Jeffrey.D.Jensen@asu.edu

1 School of Life Sciences, Arizona State University, Tempe, AZ, USA mutagenesis is also commonly noted in this regard (e.g., Bull et al. 2007), the mutational meltdown framework is in fact more general, and critically incorporates the stochastic effects inherent to natural populations (see Matuszewski et al. 2017).

While meltdown has been discussed largely in the negative context of a threat to small or endangered populations, it also has relevance in the positive context of inducing the extinction of a viral population within a patient. One drug in particular, favipiravir, has been demonstrated to inhibit the RNA-dependent RNA polymerase (RdRp) of RNA viruses (Furuta et al. 2013; Baranovich et al. 2013), and in vitro studies in influenza A virus (IAV) have specifically examined the relevance of a mutational-meltdown model in the presence of this inhibitor. Bank et al. (2016), utilizing experimental passaging at different drug concentrations, described potential viral adaptation at low-concentrations. However, at higher concentrations, mutations accumulated at a nearly linear rate until a transition point was reached, at which a sharp increase in mutational accumulation was observed, followed by population collapse. Significantly, as opposed to targeting a specific genomic region, this input of deleterious mutations is a genome-wide effect, raining deleterious variants on all functionally essential genomic regions.

Also working in IAV, Ormond et al. (2017) examined the combined effect of favipiravir with oseltamivir, a widely-used treatment with well-studied resistance mutations. A similar mutational meltdown outcome was observed, with the selective sweeps of oseltamivir-resistant mutations appearing to actually speed population decline, owing to the resulting hitchhiking of linked deleterious variants in the viral population (and see the related work of Pénnison et al. 2017).

We believe that these results at least suggest the potential therapeutic value of inducing mutational meltdown in SARS-CoV-2 patient populations. While interest in favipiravir is currently motivating clinical trials, with initial results as of March 2020 suggesting faster viral clearance 
compared to other tested drug treatments (Dong et al. 2020), in vitro studies will also be of great value to understand the interplay of mutational meltdown dynamics with the noteworthy biological differences of CoV-2 relative to the better studied IAV (Smith et al. 2013). Thus, while many key questions remain in need of exploration, results to date demonstrate the importance of this effort, and highlight the great value of utilizing population genetic theory to address such crucial public health concerns.

\section{Compliance with ethical standards}

Conflict of interest The authors declare that they have no conflict of interest.

Publisher's note Springer Nature remains neutral with regard to jurisdictional claims in published maps and institutional affiliations.

\section{References}

Bank C, Renzette N, Liu P, Matuszewski S, Shim H, Foll M, Bolon DN, Zeldovich KB, Kowalik TF, Finberg RW, Wang JP, Jensen JD (2016) An experimental evaluation of drug induced mutational meltdown as an antiviral treatment strategy. Evolution 70:2470-2484
Baranovich T, Wong SS, Armstrong J, Marjuki HJ, Webby RJ, Webster RG, Govorkova EA (2013) T-705 (favipiravir) induces lethal mutagenesis in influenza A H1N1 viruses in vitro. J Virol 87:3741-3751

Bull JJ, Sanjuan R, Wilke CO (2007) Theory of lethal mutagenesis for viruses. J Virol 81:2930-2939

Dong L, Hu S, Gao J (2020) Discovering drugs to treat coronavirus disease 2019 (COVID-19). Drug Disco Ther 14:58-60

Furuta Y, Gowen BB, Takahashi K, Shiraki K, Smee SF, Barnard DL (2013) Favipiravir (T-705), a novel viral RNA polymerase inhibitor. Antivir Res 100:446-454

Li G, De Clercq E (2020) Therapeutic options for the 2019 novel coronavirus (2019-nCoV). Nat Rev Drug Discov 19:149-150

Lynch M, Burger R, Butcher D, Gabriel W (1993) The mutational meltdown in asexual populations. J Hered 84:339-344

Lynch M, Gabriel W (1990) Mutation load and the survival of small populations. Evolution 44:1725-1737

Matuszewski S, Ormond L, Bank C, Jensen JD (2017) Two sides of the same coin: a population genetics perspective on lethal mutagenesis and mutational meltdown. Virus Evol 3:1-5

Ormond L, Liu P, Matuszewski S, Renzette N, Bank C, Zeldovich K et al. (2017) The combined effect of oseltamivir and favipiravir on influenza A virus evolution. Genome Biol Evol 9:1913-1924

Pénnison S, Singh T, Sniegowski P, Gerrish P (2017) Dynamics and fate of beneficial mutations under lineage contamination by linked deleterious mutations. Genetics 205:1305-1318

Smith EC, Blanc H, Surdel MC, Vugnuzzi M, Denison MR (2013) Coronaviruses lacking exoribonuclease activity are susceptible to lethal mutagenesis: evidence of proofreading and potential therapeutics. PLoS Path 9:e1003565 